\title{
Troublesome triad: fibrosing mediastinitis, severe pulmonary hypertension, and pulmonary vein stenosis
}

\author{
Gregory W Wigger ${ }^{* 1}$ and Jean Elwing ${ }^{2}$ \\ ${ }^{1}$ Resident Physician, Department of Internal Medicine, University of Cincinnati Medical Center, Cincinnati, OH 45267, USA \\ ${ }^{2}$ Associate Professor of Medicine, Department of Pulmonary, Critical Care, and Sleep Medicine, University of Cincinnati Medical Center, Cincinnati, OH 45267, USA
}

\begin{abstract}
Fibrosing mediastinitis is a disorder of invasive and proliferative fibrous tissue growth in the mediastinum. Its occurrence is rare and cause unknown, though it has been associated with several infections. The fibrosis of mediastinal structures, particularly vasculature, results in common symptoms of cough, dyspnea and hemoptysis due to pulmonary hypertension, pulmonary edema, and parenchyma fibrosis. Progression of disease carries a high mortality rate and causes of death are typically due to cor pulmonale,pulmonary infections and respiratory failure. Several medical treatments and surgical procedures have been unsuccessful; however the use of endovascular stenting is showing promising results. Early recognition and diagnosis are essential to provide patients with the best chance of survival and management options. We review the case of a 43 year-old female diagnosed with fibrosing mediastinitis and sequelae of pulmonary vein stenosis and pulmonary hypertension. Unfortunately, she was a poor candidate for pulmonic vein stenting due to her unstable condition. Without treatment of the stenosis, her clinical status and pulmonary hypertension worsened. Pulmonary vasodilators resulted in pulmonary edema and diuresis resulted in hypotension. Ultimately her condition was untreatable. This case illustrates the devastating effects of advanced fibrosing mediastinitis.
\end{abstract}

\section{Clinical presentation/Course}

A 43 year-old female with past medical history of hypertension, chronic thromboembolic pulmonary disease, and a remote right lower lobe lobectomy after a prior motor vehicle accident was transferred from an outside hospital where she presented with shortness of breath. The patient initially presented with several weeks of progressively worsening dyspnea on exertion. She was previously treated with antibiotics for presumed pneumonia without improvement and was transferred to our facility when a right heart catheterization showed a pulmonary arterial pressure of $118 / 64 \mathrm{mmHg}$ and a wedge pressure of $27 \mathrm{mmHg}($ Table 1).Upon arrival the patient's respiratory status rapidly worsened, eventually requiringintubation. CT pulmonary angiogram was negative for an acute pulmonary embolism but did show widespread airspace disease with superimposed edema as well as narrowing of the superior pulmonary vein and mediastinal calcifications(Figures 1-3). The patient was treated with empiric antibiotics, respiratory support, and aggressive intravenous diuresis. Her hospital course was complicated by oliguric acute tubular necrosis requiring continuous renal replacement therapy as well as hypotension requiring vasopressors and inotropic support. A cardiac CT showed severe stenosis of the left superior pulmonary vein(Figure 4), an enlarged pulmonary artery and inferior vena cava, as well as a dilated right atrium and ventricle. A thoracic CT showed calcified lymphadenopathy and bilateral hilar calcifications consistent with fibrosing mediastinitis. Despite all interventions the patient's health deteriorated. The pulmonary pressures continued to rise(Table 1). Attempts at pulmonary vasodilation with inhaled nitric oxide and sildenafil were unsuccessful due tosubsequent pulmonary edema. The patient was evaluated by interventional cardiology and cardiac surgery and deemed to have too extensive and unstable of disease for pulmonic vein stenting. She was assessed for extracorporeal membrane oxygenation but due to her irreversible condition was not a candidate.
The patient's family elected to withdraw care and the patient passed after extubation.

\section{Discussion}

Fibrosing mediastinitis (FM), also known as sclerosing mediastinitis and mediastinal fibrosis, is an uncommon disorder of invasive, proliferating fibrous tissue in the mediastinum. While the exact etiology and pathogenesis of fibrosing mediastinitis are not fully understood, many have linked the disease to Histoplasma capsulatum infection [1]. Other infectious etiologies including tuberculosis, aspergillosis and blastomycosis $[1,2]$ have been implicated.The fibrotic proliferation within the mediastinum leads to invasion and destruction of normal structures including the esophagus, trachea, and vasculature [3]. Patients who develop pulmonary artery or venous stenosis have increased morbidity and mortality [4]. Symptoms are typically due to compression or obstruction of these structures and are most commonly cough, dyspnea and hemoptysis [1,3].Chest radiographs typically show a widened mediastinum with distortion of the typical mediastinal lines. Computed tomography scans show an infiltrative, calcified mediastinal mass with either focal or diffuse fibrosis. Causes of death for patients with FM include cor pulmonale, respiratory failure and recurrent pulmonary infections $[1,3]$. There is little evidence of benefit in treatment with antifungal or corticosteroids, and trials of surgical therapies have been unsuccessful. However, the use of endovascular stenting is a growing and promising avenue for treatment.

Correspondence to: Gregory W Wigger, MD, Resident Physician, Department of Internal Medicine, University of Cincinnati Medical Center, Cincinnati, $\mathrm{OH}$ 45267, USA, E-mail: gregory.wigger@uc.edu

Key words: endovascular stent, fibrosing mediastinitis, pulmonary hypertension, pulmonary vein stenosis

Received: April 20, 2017; Accepted: May 01, 2017; Published: May 04, 2017 
Table 1. Right heart catheterization/Swan Ganz catheter measurements showing significant pulmonary hypertension as well as elevated wedge pressures secondary to pulmonary vein stenosis. In addition, the trend in ventilator settings notes the patient's progressive respiratory failure due to fibrosing Mediastinitis.

\begin{tabular}{|c|c|c|c|c|c|c|}
\hline & Outside Hospital & Day \#0 & Day \#1 & Day \#2 & Day \#4 & Day \#5 \\
\hline $\begin{array}{c}\text { Pulmonary Artery Pressure } \\
(\mathrm{mmHg})\end{array}$ & $118 / 64$ & $100 / 64$ & $107 / 42$ & $73 / 37$ & $111 / 49$ & $91 / 46$ \\
\hline $\begin{array}{l}\text { Mean Pulmonary Artery } \\
\text { Pressure (mmHg) }\end{array}$ & 73 & 69 & 66 & 52 & 70 & 62 \\
\hline $\begin{array}{c}\text { Central Venous Pressure } \\
(\mathrm{mmHg})\end{array}$ & 22 & 16 & 17 & 13 & 14 & 14 \\
\hline Cardiac Output (L/min) & 6.8 & 7.35 & 7 & 9.5 & - & - \\
\hline Cardiac Index $\left(\mathrm{L} / \mathrm{min} / \mathrm{m}^{2}\right)$ & 2.77 & 2.95 & 2.8 & 3.8 & - & - \\
\hline $\begin{array}{c}\text { Pulmonary Capillary Wedge } \\
\text { Pressure (mmHg) }\end{array}$ & 27 & 30 & - & - & - & - \\
\hline$\underset{(\mathrm{mmHg})}{\operatorname{PEEP}\left(\mathrm{cmH}_{2} \mathrm{O}\right) / \mathrm{FiO}_{2} / \mathrm{PaO}_{2}}$ & - & $16 / 1.0 / 69$ & $14 / 0.95 / 91$ & $13 / 0.5 / 81$ & $13 / 1.0 / 130$ & $15 / 0.6 / 160$ \\
\hline 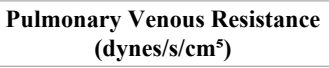 & 541 & 424 & - & - & - & - \\
\hline
\end{tabular}
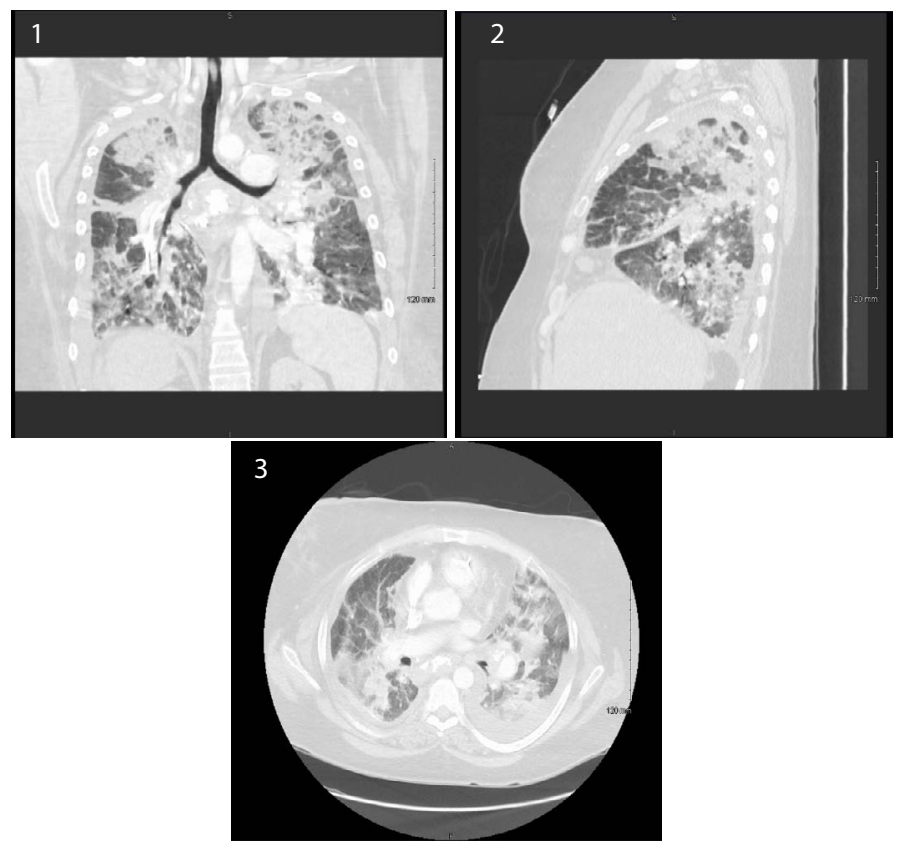

Figures 1-3. Coronal, sagittal, and axial CT imaging show the characteristic mediastinal fibrosis and calicified masses seen in fibrosing Mediastinitis.

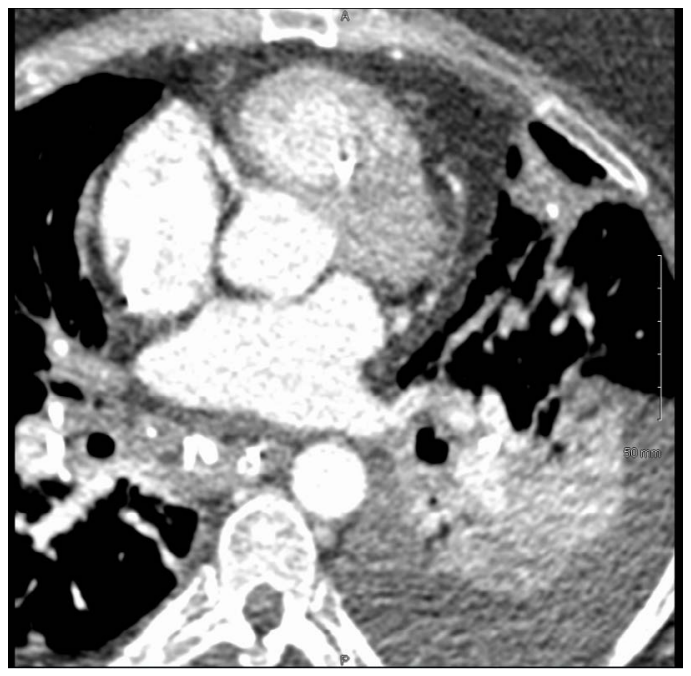

Figure 4. Cardiac CT with left superior pulmonary vein stenosis.
Several attempts have been made investigating endovascular stenting as a therapeutic intervention in FM patients with symptomatic stenosis. Several case reports of patients with symptomatic FM due to pulmonary artery stenosis have noted improvement in symptoms and disease course after pulmonary arterial stenting. Specifically results have noted improved exercise tolerance, reduction in oxygen requirement [5], complete resolution of dyspnea [6], resolution of pulmonary hypertension [7] and prolonged symptomatic relief after stent placement [8]. A comprehensive review of endovascular stenting for FM patients at a large specialized center included stenting of the superior vena cava (SVC), and both the pulmonary arteries and veins. The results include stenting of $40 \mathrm{FM}$ patients, the majority with bilateral and multi-vessel disease. Overall, $87 \%$ patients showed a clinical improvement in the range of 2-144 months post-stent. Instent restenosis occurred in $28 \%$ of patients, the majority in SVC stents. Patients who received stents showed an improvement in survival at 5 years as compared to historical data. Major complications from the procedure occurred in $10 \%$ of patients and included vessel injury, reperfusion pulmonary edema, thrombosis and stent malposition $[9,10]$. Additionalsmaller reviews of endovascular stenting of symptomatic FM patients have been done. Results showed similar immediate hemodynamic and symptomatic improvement. Looking specifically at patients with pulmonary vein stenosis, an increased rate of restenosis was noted in stents to the pulmonary vein as well as further complications of vessel damage and hemoptysis [11].Despite short-term benefit and symptomatic relief, these patients had a onemonth mortality of $37.5 \%$ and a reported restenosis rate of $50 \%$ [12].

In this case report, the patient suffered from such significant sequelae from fibrosing mediastinitis that her condition was ultimately untreatable by medication or procedural intervention. While the CT only visualized stenosis of the left superior pulmonary artery, it is the authors' opinion that the patient had additional unseen stenosis of multiple pulmonary veins given the notable pulmonary hypertension, pulmonary edema with pulmonic vasodilation, and respiratory failure. The measurements from the right heart catheterization indicate a level of obstruction that cannot be explained by a single pulmonary vein stenosis as the pulmonary and wedge pressures were severely elevated. This indicates significant obstruction to pulmonary venous outflow, a phenomenon not explainedbysmall vessel pulmonary veno-occlusive disease or pulmonary arterial hypertension. Fibrosing mediastinitis led to the invasive fibrosis and later stenosis of the pulmonary veins resulting in post-capillary pulmonary hypertension and subsequentpulmonary edema. As discussed, there are no viablemedical 
or surgical interventionsfor FM and the patient was too unstable for a stenting procedure. Without treatment of the stenosis, any pulmonic vasodilation for the pulmonary hypertension resulted in pulmonary edema and worsening respiratory distress. Subsequent diuresis or continuous renal replacement therapy for the pulmonary edema resulted in hypovolemia and hypotension. Ultimately making this troublesomeclinical triad of fibrosing mediastinitis, severe pulmonary hypertension, and pulmonary vein stensosis an untreatable condition.

This case illustrates the devastating sequelae of fibrosing mediastinitis. Early recognition and diagnosis are essential to provide patients with the best chance of survival and management options. If diagnosed late, treatment options become severely limited.Studies show a promising future for endovascular stenting for the treatment of patient with vascular stenosis. Further trials of different stent sizes, placement, prototypes, and concurrent medical therapy are needed to improve strategies for pulmonary vein stenting.

\section{Authorship and contribution}

Authors: Gregory W Wigger MD, Jean Elwing MD.

\section{References}

1. Rossi SE, McAdams HP, Rosado-de-Christenson ML, Franks TJ, Galvin JR (2001) Fibrosing mediastinitis. Radiographics 21: 737-757. [Crossref]

2. Arbra CA, Valentino JD, Martin JT (2015) Vascular sequelae of mediastinal fibrosis Asian Cardiovasc Thorac Ann 23: 36-41. [Crossref]
3. Loyd JE, Tillman BF, Atkinson JB, Des Prez RM (1988) Mediastinal fibrosis complicating histoplasmosis. Medicine (Baltimore) 67: 295-310. [Crossref]

4. Berry DF, Buccigrossi D, Peabody J, Peterson KL, Moser KM (1986) Pulmonary vascular occlusion and fibrosing mediastinitis. Chest 89: 296-301. [Crossref]

5. Fontaine AB, Borsa JJ, Hoffer EK, Bloch RD, So C (2001) Stent placement in the treatment of pulmonary artery stenosis secondary to fibrosing mediastinitis. $J$ Vasc Interv Radiol 12: 1107-1111. [Crossref]

6. Guerrero A, Hoffer EK, Hudson L, Schuler P, Karmy-Jones R (2001) Treatment of pulmonary artery compression due to fibrous mediastinitis with endovascular stent placement. Chest 119: 966-968. [Crossref]

7. Kandzari DE, Warner JJ, O'Laughlin MP, Harrison JK (2000) Percutaneous stenting of right pulmonary artery stenosis in fibrosing mediastinitis. Catheter Cardiovasc Interv 49: 321-324. [Crossref]

8. Satpathy R, Aguila V, Mohiuddin SM, Khan IA (2007) Fibrosing Mediastiniti Presenting as Pulmonary Stenosis: Stenting Works. Int J Cardiol 118: 85-86. [Crossref]

9. Doyle TP, Loyd JE, Robbins IM (2001) Percutaneous pulmonary artery and vein stenting: a novel treatment for mediastinal fibrosis. Am J Respir Crit Care Med 164: 657-660. [Crossref]

10. Albers EL, Pugh ME, Hill KD, Wang L, Loyd JE, Doyle TP (2011) Percutaneous vascular stent implantation as treatment for central vascular obstruction due to fibrosing mediastinitis. Circulation 123: 1391-1399. [Crossref]

11. Ferguson ME, Cabalka AK, Cetta F, Hagler DJ (2010) Results of Intravascular Stent placement for Fibrosing Mediastinitis. Congenit Heart Dis 5: 124-133. [Crossref]

12. Ponamgi SP, DeSimone CV, Lenz CJ, Coylewright M, Asirvatham SJ, et al. (2015) Catheter-based intervention for pulmonary vein stenosis due to fibrosing mediastinitis: The mayo clinic experience. Int J Cardiol Heart Vasc 8: 103-107. [Crossref]

Copyright: C2017 Wigger GW. This is an open-access article distributed under the terms of the Creative Commons Attribution License, which permits unrestricted use, distribution, and reproduction in any medium, provided the original author and source are credited. 